\title{
Phylogenetic Analysis
}

National Cancer Institute

\section{Source}

National Cancer Institute. Phylogenetic Analysis. NCI Thesaurus. Code C18940.

Phylogenetic analysis is the study of evolutionary relationships among the many different kinds of life on earth, both living (extant) and dead (extinct). It includes creating evolutionary trees using computer programs. 